\title{
Unresponsive patient after flexible bronchoscopy A fatal case of cerebral air embolism
}

I. Rebelo, S. Mauricio, C. Madeira, F. Resende - Instituto Português de Oncologia de Lisboa, Portugal (s.duquemauricio@gmail.com)

\section{BACKGROUND}

Vascular air embolism (VAE) during bronchoscopy is a rare but serious complication. Even though considered a safe procedure, bronchoscopy implicates several risk factors for VAE:

Sitting position • Pressure gradient • Air inflation
Clinical presentation depends on the volume and rate of gas infusion, and can range from clinically undetectable to cardiovascular collapse.

Neurological manifestations may arise from arterial or paroxysmal emboli, or be secondary to hypoperfusion.

\section{CASE REPORT}

19 yo female, stage IV Hodgkin Iymphoma with lung spread(1)

Flexible bronchoscopy under sedation with propofol:

after lung biopsy, air instillation $(120 \mathrm{~mL})$ and lavage with saline
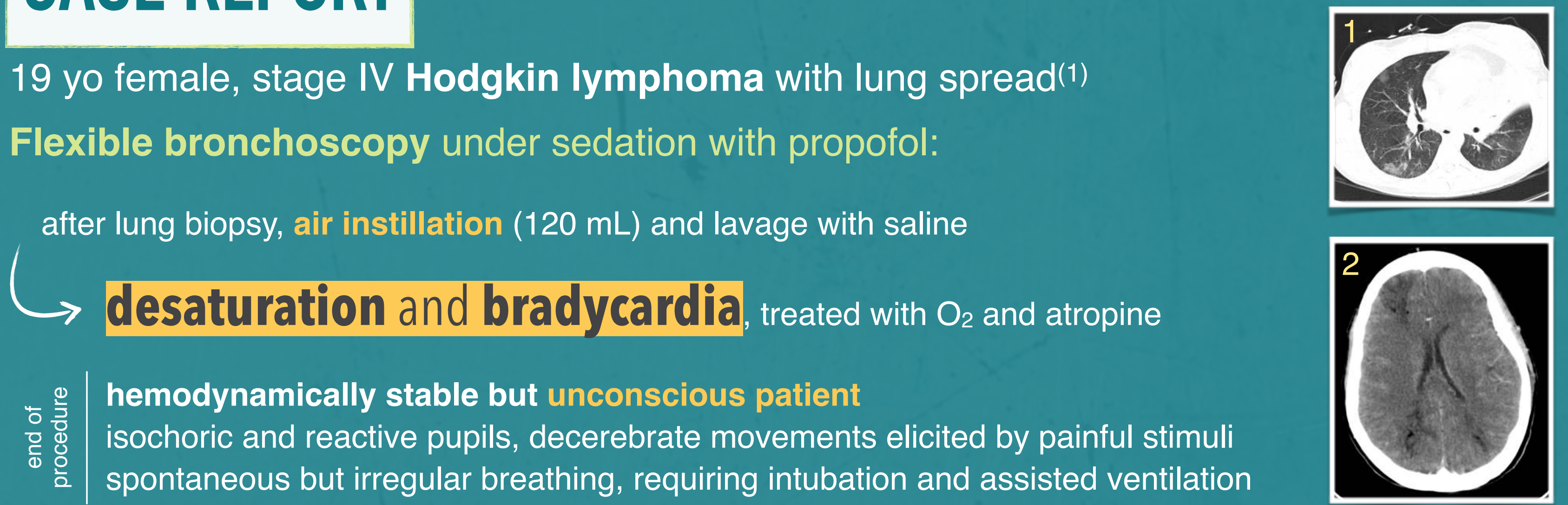

Brain CT: cortical air emboli and multiple infarctions ${ }^{(2)}$

Hyperbaric oxygen therapy (HBOT) initiated $8 \mathrm{~h}$ after the event, repeated the next day Neurological deterioration with diffuse cerebral edema(3), death at day 3

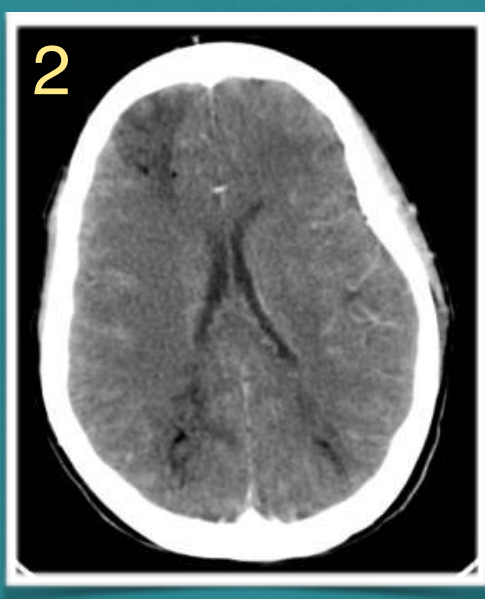

Blood gas analysis: normal

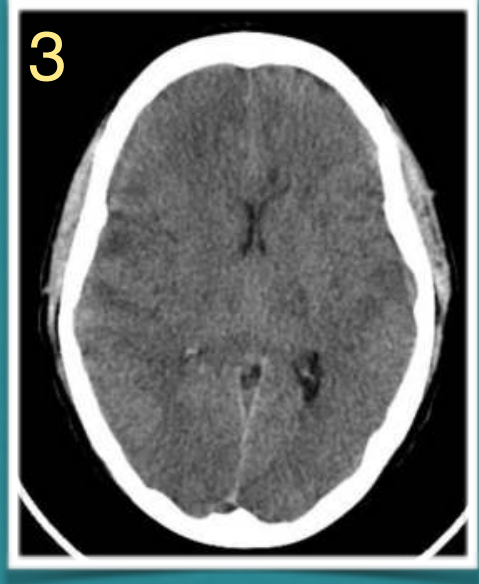

\section{DISCUSSION}
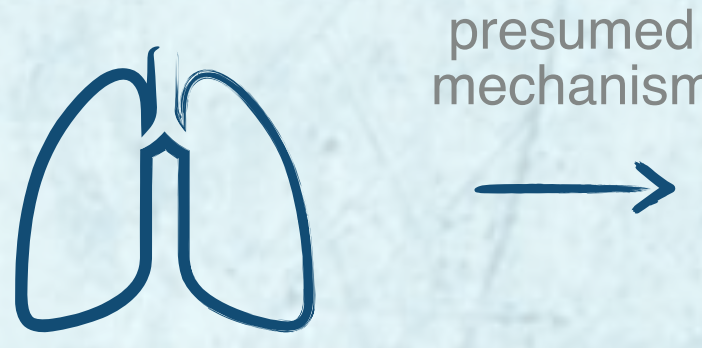
mechanism

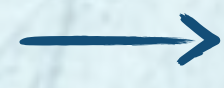

high bronchial pressure and trauma to a necrotic lesion leading to air entering pulmonary veins...
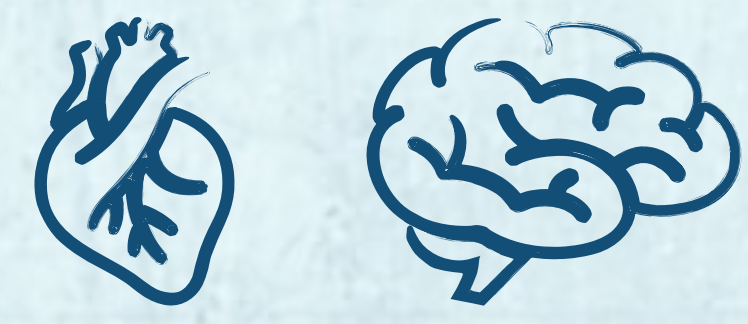

... left heart and cerebral vessels

clinically stable sedated patient: neurological signs recognized only at the end of the procedure
- high level of suspicion is crucial for early recognition and prevention of further air entry

- a drop in $\mathrm{ETCO}_{2}$ may be an early sign, but precordial doppler is more sensitive

- likely benefit for HBOT in the $1^{\text {st }}$ few hours (high $\mathrm{O}_{2}$ blood content reduces size of air emboli and ischaemia), however brain infarcts on $1^{\text {st }}$ CT carried a poor prognosis or present focal signs or seizures following bronchoscopy

risk assessment, monitoring and prevention strategies are needed 